\title{
émulations
}

\section{Ce que perdre un·e enfant fait à la famille}

\section{Le deuil comme marge et frontière de la parentalité}

\author{
Lucie Jégat
}

Émulations - Revue de sciences sociales

2019, n³2, «Aux frontières de la parenté. Un éclairage par les marges ».

\section{Article disponible à l'adresse suivante}

https://ojs.uclouvain.be/index.php/emulations/article/view/jegat

\section{Pour citer cet article}

Lucie Jégat, « Ce que perdre un·e enfant fait à la famille. Le deuil comme marge et frontière de la parentalité », Émulations, n³2, Mise en ligne le 20 avril 2020.

DOI : 10.14428/emulations.032.05

Distribution électronique : Université catholique de Louvain (Belgique) : ojs.uclouvain.be

(C) Cet article est mis à disposition selon les termes de la Licence Creative Commons Attribution, Pas d'Utilisation Commerciale 4.0 International. http://creativecommons.org/licenses/by-nc/4.0/

Éditeur : Émulations - Revue de sciences sociales / Presses universitaires de Louvain https://ojs.uclouvain.be/index.php/emulations

ISSN électronique : 1784-5734

\section{PUL PRESSES UNIVERSITAIRES




\title{
Ce que perdre un·e enfant fait à la famille
}

\author{
Le deuil comme marge et frontière de la parentalité
}

Lucie Jégat ${ }^{1}$

\begin{abstract}
[Résumé] Cet article se propose d'étudier, dans le contexte français, ce que la perte d'un enfant fait à la famille et à ceux qui la constituent, et ce à travers le deuil particulier d'un·e enfant adolescent·e. En s'appuyant sur des entretiens réalisés avec des parents ainsi qu'avec des frères et sœurs ayant perdu un·e enfant (ou un membre de la fratrie) âgé·e de 12 à 25 ans, cet article tend à montrer que le deuil produit une marginalité particulière, résultant de la confrontation entre deux statuts contradictoires. En mobilisant les notions de « frontières » et de «marge » dans une perspective contemporaine et sociologique, il s'agit de montrer que, si les parents endeuillé'e-s se retrouvent dans une situation marginale et cherchent à réaffirmer un rôle parental remis en cause par le deuil, cette position ne peut être comprise qu'en relation avec celle de l'enfant décédé·e et de la place qui lui est désormais accordée dans la famille. Les modalités de la reconfiguration familiale et de la redéfinition de ses frontières sont ainsi analysées.
\end{abstract}

Mots-clés : deuil, famille, parentalité, marginalité.

What losing a child does to the family: Grief and bereavement as a margin and frontier of parenting

[Abstract] This article aims to study the consequences of the loss of a child on the family and its members, through the particular example of children who died during their adolescence. Based on interviews with parents and siblings having lost a child (or sibling) aged 12 to 25 , this article suggests that bereavement produces a particular marginality, resulting from the confrontation between two contradictory statuses. We will use the notions of "boundaries" and "margins" in a contemporary and sociological perspective to show that bereaved parents find themselves in a marginal situation and seek to reaffirm their parental role, which is called into question by bereavement. However, their position can only be understood in relation to that of the deceased child and his or her place in the family. The modalities of family reconfiguration and the redefinition of its borders will therefore be analysed.

Keywords: bereavement, family, parenthood, marginality.

\section{Introduction}

Lorsque l'on cherche à définir le deuil de nos jours, la définition anthropologique classique, telle qu'elle est donnée par Arnold Van Gennep (2011), semble toujours opératoire : le deuil est défini comme un état de marge pour les personnes survivantes, elles y entrent par les rites de séparation et elles en sortent par des rites de réintégration ${ }^{2}$. Entre les deux, elles seraient, dans cette perspective anthropologique, maintenues dans une forme de « marginalité » transitoire, car restreinte temporellement. Cependant, le

\footnotetext{
${ }^{1}$ Université Paul Valéry Montpellier 3, Centre Max Weber, Lyon.

2 Je remercie chaleureusement Corentin Roquebert et Vanina Mozziconacci pour leurs relectures et commentaires qui ont permis d'améliorer significativement ce texte.
} 
développement de la thèse du « tabou » de la mort dans les années 1970 vient modifier les formes de cette " marginalité » (Ariès, 1977). L’hypothèse alors développée est que la ritualité contemporaine autour de la mort, conçue comme minimale, rendrait les deuils « impossibles » (Jeudy, Baudry, 2001). Dans l'idée d'un tabou de la mort, l'absence de ritualité rallongerait la sortie de la situation de "marginalité », et ce d'autant plus que le deuil concerné est perçu comme exceptionnel. Ces travaux ont cependant peu été poursuivis en France depuis les années 1980 et ce n’est que très récemment que quelques jeunes chercheur·e·s ont commencé à s'intéresser à ce qui pourrait constituer une sociologie du deuil (Julier-Costes, 2013 ; Roudaut, 2012). Si Martin Julier-Costes (2012) s'intéresse à des ritualités funéraires singulières à l'occasion du décès d'un jeune, Karine Roudaut (2013) pour sa part analyse le deuil de façon plus générique comme processus de socialisation/désocialisation. Cependant, aucun·e de ces deux chercheur·e·s ne pose la question de la façon dont le deuil vient modifier la structure familiale et les rôles parentaux assignés. Nous nous proposons donc ici de revisiter les concepts de " marges » et de " frontières » dans des approches plus contemporaines, en centrant l'analyse sur la famille et la façon dont un deuil particulier, celui d'un·e enfant adolescent·e, vient modifier à la fois la parentalité ${ }^{3}$ et les frontières familiales.

La configuration familiale marquée par la perte d'un·e enfant, et notamment d'un·e enfant " adolescent.e $»^{4}$, interpelle de par son exceptionnalité statistique : les décès d'enfants de moins de 20 ans représentent 0,8 \% de l'ensemble des décès en France en $2017^{5}$. On peut donc faire l'hypothèse que les parents en deuil d'enfant sont dans une situation d'autant plus « marginale » qu'elle est statistiquement exceptionnelle. Nous verrons dans une première partie que les parents endeuilléee-s sont dans une position que l'on peut qualifier de « liminale » qui les pousse cependant à réaffirmer leur statut de parent. Mais cette liminalité ne peut se comprendre sans analyser la position de l'enfant défunt.e par rapport au groupe familial, position qui oblige à une redéfinition permanente des frontières familiales, ce que nous verrons dans un second temps.

Cet article se fonde sur une recherche doctorale en cours portant sur les trajectoires individuelles et familiales après le décès d’un·e enfant âgé.e de 12 à 25 ans. Le choix de cette tranche d'âge repose sur un double argument sociologique et démographique. D’un point de vue démographique, l'entrée dans l'adolescence marque le début d'un pic de mortalité pour les enfants, et notamment pour les jeunes garçons ${ }^{6}$ (du fait, notam-

\footnotetext{
${ }^{3}$ Pour plus de clarté, le terme de «parenté » sera utilisé pour désigner la configuration familiale en général, et celui de « parentalité » pour désigner le statut parental et le travail parental qui l'accompagne.

${ }^{4}$ Aussi arbitraire que soit la désignation d'« adolescent·e » pour une classe d'âge aussi hétérogène que les 12-25 ans, elle permet de mettre l'accent sur l'autonomisation progressive des enfants de cet âge (Van de Velde, 2015) : 12 ans pour l'entrée au collège et l'intensification de la socialisation secondaire, et 25 ans pour reprendre les critères d'émancipation familiale et de possibilité de demande du Revenu de solidarité active (RSA) (Lima, 2015).

${ }^{5}$ D'après la base de données État civil, 2017 de l'INSEE (disponible sur le site de l'INSEE). Calculs de l'autrice.

${ }^{6}$ Pour l'année 2017, sur l'ensemble des enfants décédé·e·s entre 12 et 25 ans, 29,1 \% étaient des filles et 70,9 \% étaient des garçons, les écarts se creusant avec l'âge (sur l'ensemble des enfants dé-
} 
ment de l'augmentation des causes accidentelles). D'un point de vue plus sociologique, l'adolescence et l'entrée dans la vie adulte marquent les débuts de la décohabitation. La tranche d'âge choisie permet donc de comparer des parcours familiaux endeuillés entre familles cohabitantes ou décohabitantes. Cette enquête s'appuie sur 41 entretiens biographiques qui ont été réalisés entre 2014 et 2017, avec des parents mais également des frères et sœurs d'enfants décédé·e·s. Dans cet échantillon, 16 entretiens ont été réalisés avec des mères, 10 avec des pères, 8 avec des sœurs et 7 avec des frères endeuillé $\cdot e \cdot s^{7}$. Ces entretiens ont été menés entre deux et dix ans après la date du décès et se sont déroulés pour la plupart au domicile familial en face à face. Le recrutement des enquêté·e·s a été fait dans la métropole lyonnaise et dans un contexte plus rural, dans les Pyrénées-Atlantiques. Les familles concernées sont des familles nucléaires, qui s'articulent donc autour d'un couple parental hétérosexuel ${ }^{8}$, même si ce couple peut s'être séparé, soit avant, soit après le décès. L’intérêt porté aux trajectoires des frères et sœurs endeuillé·e·s a plutôt conduit à un recrutement de familles où l'enfant défunt·e n'était pas enfant unique. Les causes de décès étant primordiales pour comprendre les trajectoires créées par le deuil, une attention particulière a été accordée à leur variété9, en excluant toutefois du recrutement les décès ayant une cause criminelle.

\section{Les parents en deuil d'enfant : une parentalité en marge}

\subsection{Un statut paradoxal}

Le statut de parent endeuilléee est paradoxal : défini, dans un premier temps, par la présence d'un·e (ou plusieurs) enfant, ce statut doit être maintenu malgré l'absence d'un.e d'entre eux. C'est donc la dimension relationnelle du statut parental qui est mise à mal dans le deuil. Si la parentalité est actuellement un champ d'études fécond et si l'entrée dans ce statut et ses modalités sont largement documentées (Oria, Camus, 2012 ; Régnier-Loilier, Perron, 2016), sa sortie, elle, n’est pas pensée. Alors que son accès n'est pas garanti (Clavandier et al., 2018 ; Giraud, 2015), en sortir semble impossible, du moins impensable. En atteste le fait qu'il n'existe aucun terme pour désigner ce statut, hormis quelques usages profanes du terme « orphelins d'enfants » (Le Galès, 2017). Comment comprendre cette absence de dénomination ? La rareté des situations ne

cédé·e·s à 14 ans, 37,3 \% étaient des filles et 62,7 \% des garçons et, à 20 ans, 25,4 \% des défunt.e.s étaient des filles et 74,6 \% des garçons). D'après la base de données État civil, 2017 de l'INSEE (disponible sur le site de l'INSEE). Calculs de l'autrice.

${ }^{7}$ Dans la mesure du possible, les entretiens ont été réalisés aussi bien avec les deux parents qu'avec les membres de la fratrie. Mais la difficulté du thème abordé a conduit à ce que certaines familles ne soient représentées que par un seul membre.

${ }^{8}$ Ce n'était pas un choix méthodologique à priori mais la rareté des familles homoparentales, liée à la rareté des décès d'enfants de cette tranche d'âge, réduit fortement la probabilité de recrutement de cette configuration familiale.

${ }^{9}$ Les causes de décès, relativement variées lors du recrutement (suicides, cancers et accidents de la route généralement), sont peu mobilisées dans ce texte. En effet, si les causes de décès introduisent une différence, c'est bien plus sur la temporalité spécifique du deuil (est-ce qu'il commence avant ou après le décès de l'enfant) que sur les pratiques parentales. 
suffit pas à expliquer l'absence de terme spécifique. Une hypothèse permettant de l'expliquer viendrait de la non-nécessité juridique de définir un nouveau statut. En effet, les statuts de veuf.ve·s ou d'orphelin.e.s ouvrent des droits spécifiques, et notamment des droits de succession. La dimension successorale paraît ainsi secondaire pour les parents endeuillée.s. Des travaux contemporains questionnent cette absence de nomination, et Anne-Sophie Giraud développe la notion de " péri-parents » (Giraud, 2015) pour désigner des cas de décès périnataux. Mais ce terme ne peut convenir pour les situations rencontrées : les péri-parents sont des parents en puissance qui n'ont pas pu atteindre la parentalité (comme statut, mais également comme ensemble de pratiques quotidiennes) du fait de la mort du fœtus. Or, les parents en deuil d'enfants adolescent·e.s ont été parents, mais l'absence d'un·e de leur enfant questionne la forme que peut prendre à présent leur statut parental. La question qui se pose est donc celle de la conciliation du deuil avec le statut de parent.

En français, la définition du deuil se heurte à l'ambiguïté et à la polysémie du terme. Sur ce point, les termes anglais apportent bien plus de précisions : ils permettent de faire la distinction entre le deuil comme sentiment (grief, ou plus spécifiquement mourning) et le deuil comme statut (bereavement) (Bacqué, Hanus, 2009). Afin de rendre compte de cette dernière acception, nous utiliserons les termes de « deuilleurs et deuilleuses " pour définir les personnes ayant perdu un proche (enfant ou membre de la fratrie). Ces termes ont comme intérêt de «souligner le travail opéré par les proches au moment du décès d'un être cher » (Julliard, Georges, 2018 : 100). Ils renvoient ainsi à la fois à un statut (être en deuil) et à une mise en activité relative à ce statut (regarder des photos, visiter le cimetière, etc.). L'utilisation de ce terme permet d'employer le terme de « deuil » autrement qu'à travers un prisme uniquement émotionnel et transitoire (qui serait consubstantiel aux représentations associées au « travail de deuil », voir Memmi, 2014). C’est donc bien l'ambiguïté statutaire qui est ici analysée : comment concilier deux statuts apparemment antagonistes, celui de parents et celui de deuilleur.se's ?

Les difficultés face à ce deuil particulier ne peuvent pas être détachées de la place de l'enfant dans la définition de l'identité parentale et du processus de « sacralisation de l'enfant » que connaissent nos sociétés contemporaines (Déchaux, 2014 ; Zelizer, 1994). De plus, à la différence de situations de parentalité empêchée ou séparée, le statut de parent en deuil d'enfant se caractérise par sa nature irrémédiable. En ce sens, le décès de l'enfant est bien une rupture biographique au sens où l'entend Michel Grossetti (2009) : il cumule imprévisibilité et irréversibilité, là où d'autres expériences de séparation peuvent toujours être conçues, du moins hypothétiquement, comme limitées temporellement. Le statut de parent deuilleur.se est donc particulier à deux égards : il est irrémédiable et advient après un temps relativement long de vie partagée. Il y a donc bien une rupture dans le statut parental avant et après le décès de l'enfant. En ce sens, le terme de " marge " peut être utilisé, non dans le sens de Van Gennep, pour désigner un statut d'entre-deux entre le monde sacré et le monde profane, mais bien pour rendre compte d'une « mise à la marge » de la parentalité, c'est-à-dire d'une 
position entre parent et non-parent. Plus tout à fait parents mais toujours défini-e.s ainsi, la question qui se pose alors est de savoir quel statut peuvent avoir ces parents endeuillé·e.s et ce que ce statut dit des relations avec l'enfant défunt·e.

\subsection{Une parentalité liminale}

Analyser la position des parents endeuilléee-s revient à analyser la forme particulière de la marginalité qui les caractérise. Plus que de marge alors, il conviendrait de reprendre la terminologie plus précise de Victor Turner (1969) et de parler ici de « liminalité ». Cette notion permet d'explorer des processus, des espaces de transitions entre les normes sociales et les normes institutionnelles et la façon dont les individus tentent, ou non, de s'y réinscrire. Or, sur ce terrain particulier, les endeuilléee-s tentent, par leurs pratiques, de réaffirmer leur appartenance au statut parental, notamment en en performant le rôle vis-à-vis de l'enfant décédé·e.

Dans les entretiens, les parents expriment de manière extrêmement claire un ressenti de liminalité : ils et elles sont toujours parents, mais des parents à part. D’après Victor Turner (1969), l'individu en position liminale présente des traits spécifiques : il échappe aux classements sociologiques puisqu'il est dans une situation d'entre-deux ; il est mort au pays des vivants.

C'est-à-dire que, de mars à janvier je ne pouvais plus vivre. Je n'étais absolument pas suicidaire, mais j'étais incapable de vivre. [...] Et je voyais vraiment pas ce que je faisais là. J'étais comme derrière une vitre. Je voyais les autres vivre. J'étais une martienne. Je savais pas comment j'allais continuer. (Françoise Laval'10, fils décédé à 12 ans en 2006 d'un cancer, entretien réalisé en 2013)

Les images de séparation (« martienne », « derrière une vitre ») évoquent bien ce détachement du monde des « vivants », c'est-à-dire ici des personnes non endeuilléee-s. Cet extrait d'entretien offre un exemple d'expression de la liminalité, qui se retrouve dans les discours ou dans les pratiques (isolement, arrêt des activités sociales par peur de «mal faire ») de la plupart des parents enquêtée.es : leur situation semble alors indéfinie et indéfinissable ; elle correspond presque à une mort sociale, une forme de «limbes » (Turner, 1969). Ici s’ajoute également la représentation du deuil comme excluante et marginalisante :

C'est-à-dire que j'étais devenue un monstre. Et je me disais « tu es en train de devenir folle », en fait « tu hantes la maison, tu n'es pas une mère normale pour tes enfants, tu peux plus vivre, tu fais tout ce que tu peux, mais tu n'y arrives pas, en même temps tu n'es pas suicidaire, mais tu n'y arrives pas. Donc tu deviens assez monstrueuse. Y a plus d'humanité ». (Françoise Laval, fils décédé à 12 ans d'un cancer)

Les deux extraits d'entretien de cette même enquêtée permettent à la fois de rendre compte de cette liminalité et d'illustrer les raisons même de cette exclusion : elle tient

\footnotetext{
${ }^{10}$ Tous les prénoms et tous les noms ont été anonymisés, en utilisant notamment l'application développée par Julien Barnier (https://data.nozav.org/app/prenoms/) permettant de retrouver les prénoms les plus donnés dans l'année de naissance des enquêté·e·s. Les noms de famille ont été modifiés de sorte à conserver des sonorités ou consonances assez proches des noms originaux.
} 
à l'ambiguïté et l'anormalité statutaire ainsi conférée (" plus une mère normale »). Le deuil d'enfant est exprimé comme une exclusion par rapport au statut parental, car il devient impossible pour cette mère de jouer son " rôle » envers les enfants encore vivant.e.s et, en même temps, de vivre pleinement la douleur de son deuil. Les figures de marginalité mobilisées dans le discours (" martienne », " monstre ») rendent bien compte à la fois de la souffrance et du sentiment d'exclusion créés par cette souffrance. C'est par la souffrance que la rupture se fait entre les autres parents et les parents en deuil.

À bien des égards, la situation des parents endeuillé·e·s peut être rapprochée de celle décrite par Henri-Jaques Stiker (2007) à propos des personnes en situation de handicap. D’après Stiker, « la différence avec la liminalité et la position de seuil, constatée par l'anthropologue dans nombre de sociétés, c'est que les personnes handicapées sont condamnées à demeurer dans l'entre-deux. [...] Il y a bien eu séparation du monde "ordinaire”, il y a bien seuil, mais la nouvelle agrégation ne se fait pas, ou si mal [...]. Il y a cristallisation, gel, de la situation intermédiaire »(Stiker, $2007: 10$ ). Le point commun entre la position des parents en deuil et celle des personnes handicapées se fait donc autour de la conception de la liminalité, qui n'est pas une liminalité temporaire, mais une liminalité qui dure, cristallisée et inscrite dans une temporalité longue.

Cette inscription dans le long terme s'accompagne également d'incertitudes normatives autour des pratiques " convenables » : la non-appartenance à la parentalité « normale » ne se voit jamais aussi bien que dans les doutes des parents sur ce qu'il « convient » de faire dans leur situation. Cela est particulièrement bien illustré par le discours de Corinne.

J'avais l'impression que j'avais plus le droit de sortir, que j'avais plus le droit d'aller au restaurant, que j'avais plus... Parce que j'avais peur du regard des gens quoi. [...] Et souvent on m'a dit « on sait pas comment tu fais parce que nous on pourrait pas». Alors je me dis, mais je disais à la psy «mais je, je suis pas normale. Je dois pas être normale quoi ». (Corinne Génu, deux filles de 16 et 18 ans décédées dans le même accident de voiture en 2013, entretien réalisé en 2015)

Du fait de l'absence de normes claires, l'idée de " continuer » à vivre s'accompagne d'une crainte du regard d'autrui : les parents endeuilléee.s devraient à la fois ne pas être trop visibles dans l'espace public, et en même temps exprimer la tristesse qui correspond à leur situation. Ce paradoxe rend bien compte de l'impossibilité de cette condition. Cette liminalité est d'autant plus difficile qu'elle dure et s'installe dans le temps. Il s'agirait ici bien d'une autre forme de parentalité, qui serait une parentalité liminale, et qu'il faudrait alors performer.

\subsection{Performer la parentalité}

Les parents en deuil voient leur statut parental questionné par l'absence d'(un·e) enfant. Afin de consolider ce statut « malmené », ils et elles tendent à le réaffirmer par des pratiques spécifiques à la situation de deuil. En ce sens, il s'agit bien pour eux et elles de " performer » la parentalité. Cette " performance » ne semble pas être propre à ce 
deuil particulier. Anne-Sophie Giraud (2015) montre ainsi que les femmes ayant perdu un·e enfant au moment de la naissance vont continuer à exercer certaines fonctions parentales telles que des démonstrations d'affection ou un entretien plus physique qui passe par le soin de la tombe ou la célébration des anniversaires. On constate des comportements similaires du côté des parents, et notamment des mères, ayant perdu un·e enfant adolescent·e. Mais, à la différence des situations d'enfants né·e·s sans vie, les parents sont ici moins à la recherche d'un statut qu'à une réaffirmation de celui-ci. En outre, cette réaffirmation suit une logique genrée : les pères et les mères endeuillé·e·s ne performent pas leur parentalité de la même manière.

Du côté maternel, la réaffirmation statutaire passe par un soin quotidien accordé aux traces de l'enfant défunt.e. L'entretien et la visite de la tombe sont en effet des pratiques courantes, voire quotidiennes, pour la plupart des mères enquêtées. Par exemple, Chantal Giovanni, dont le fils est décédé à 20 ans d'un cancer, explique qu'elle s'y rendait tous les jours, deux à trois fois par jour, et ce même plusieurs années après la mort de son fils. De manière analogue, Magdalena Therreau (fils de 25 ans décédé à l'étranger d'un accident de voiture) a tenu à ce que son fils soit enterré dans sa commune afin d'aller le voir tous les jours, et ce malgré le désir de son mari de mettre le corps dans la concession familiale, située dans une ville voisine ${ }^{11}$. L'attention à la proximité physique et les fréquences des visites illustrent cette volonté d'apporter un soin, une attention, de la même manière que cela pourrait être fait pour un'e enfant encore en vie. L'entretien et la conservation des chambres à coucher peuvent également être analysés comme une manière de se maintenir dans un rôle de soin vis-à-vis de l'enfant. Les quelques chambres qu'il m'a été donné de voir n'avaient pas été touchées, malgré le temps écoulé depuis le décès (parfois plus de dix ans). Or, le souci accordé à ces espaces malgré leur non-utilisation (les mères concernées me disent y faire régulièrement la poussière, y changent les draps, etc.) permet d'inscrire la maternité endeuillée dans des pratiques de soin quotidien, et ce malgré l'absence de la personne à qui ces soins sont destinés. L'activité de soin parental permet donc de continuer la parentalitéét.

Si nous insistons ici sur les pratiques maternelles, ce n'est pas pour montrer que l'ambiguïté en termes de rôle ne joue que pour elles. Au contraire, les pères mettent également en place des pratiques spécifiques. Cependant ces pratiques sont moins tournées vers les discours ou les « soins » et davantage vers la mise en activité, soit professionnelle soit manuelle. C'est ce dont rend compte Claude Therreau (fils décédé à 25 ans décédé d'un accident de voiture) lorsqu'il explique avoir mis en place un atelier d'activités manuelles pour faire venir les pères endeuillés : «Et en tenant un outil, en faisant des choses ensemble, on peut parler, on peut échanger, on peut... Mais se retrouver autour d'une table pour discuter, alors là, pas du tout, pas du tout. Mais autour d'une activité

${ }^{11}$ Cette différence genrée renvoie à une logique d'inscription dans la lignée mise en avant par certains pères, mais jamais évoquée par les mères (Jégat, 2015).

12 Il faut toutefois noter que s'il n'y a pas, chez les parents rencontré·e·s, de non-expression d'un registre de quotidienneté dans la relation avec l'enfant défunt·e, cela provient également d'un biais méthodologique : l'entretien est, dès le départ, négocié et centré sur le deuil d'enfant. La relation avec l'enfant disparu·e est donc au centre des discours recueillis. 
manuelle oui ». Cette observation tend à souligner le fait que le deuil, loin de remettre en cause les normes de genre, tend au contraire à les renforcer dans leurs dimensions les plus traditionnelles (Jégat, 2019). Dans le même ordre d’idée, les visites au cimetière me sont davantage racontées par les mères que par les pères en deuil.

Cette segmentation genrée s'accompagne également d'un " travail émotionnel » (Hochschild, 2017) plus fort du côté des mères, les pères leur déléguant, la plupart du temps, les activités d'expression des émotions (Jonas, 2006). Le propre des pratiques des mères (pour la majorité de celles rencontrées) est de s'inscrire dans un registre de quotidienneté : il faut que l'enfant reste une « contrainte » temporelle. C'est la dimension quotidienne et contraignante qui renvoie à la responsabilité maternelle et permet de perpétuer le rôle maternel malgré l'absence de l'enfant. Si la dimension quotidienne est importante dans les discours paternels, elle repose alors davantage sur une expression plus symbolique (voir infra).

Cependant, si les pratiques paternelles et maternelles après le décès sont différenciées, elles n'en tendent pas moins, sous des modalités différentes, à réaffirmer un statut parental : les parents, pères et mères, endeuillée-s se retrouvent dans la liminalité de leur situation par rapport aux autres parents. Mais cette position marginale des parents endeuillée.es ne peut se comprendre sans analyser le réseau de relations dans laquelle elle s'institue, de même que l'ambiguïté statutaire des parents en deuil ne peut se comprendre que dans la relation avec l'enfant défunt·e. Or, l'absence de l'enfant ne met pas fin à la relation. Ainsi la dimension liminale du statut des parents découle de l'incertitude autour de la place de l'enfant décédée ; se retrouvent alors des mécanismes davantage analysés par l'anthropologie, qui mobilisent notamment la peur de la contamination symbolique.

\subsection{Deuil et contamination symbolique}

La position liminale des parents endeuillée.es n'est pas seulement visible dans leur rôle parental, elle l'est aussi du fait de l'exclusion qu'ils et elles connaissent de leurs anciennes sphères de sociabilité. Ainsi, la mise à l'écart est explicitée dans la distance qui se crée entre eux et les personnes qui forment et formaient leur entourage. Après le décès de l'enfant, le constat d'un « tri », notamment amical, est l'expérience la plus partagée dans nos entretiens :

[...] Je crois encore une fois chacun fait comme il peut, avec ce qu'il a et certains sont effrayés. Il y a la peur de la contagion. Probablement [...]. Je crois qu'il y a un peu la peur de la contagion. Quelque chose qui est contagieux. À trop côtoyer le drame, on risque d'être contaminé. Donc c'est vrai que oui, y a un tri. (Claude Therreau, fils de 25 ans décédé à l'étranger d'un accident de voiture en 2006, entretien réalisé en 2014)

Cette expérience du « tri » renvoie à une double représentation classique autour du deuil et de la souffrance. Représentation anthropologique d'abord, avec l'idée d'exclusion temporaire des deuilleur-se·s (Gennep, 2011 ; Hertz, 1906) mais également représentation plus philosophique, celle d'une souffrance inexprimable donc excluante 
(Ricoeur, 2013). Ces deux éléments se combinent et se retrouvent dans les discours pour traduire l'expérience de marginalisation telle qu'elle est vécue par les deuilleur·se·s. Ils produisent ainsi une peur de la contagion, vecteur de marginalisation. Cette dimension a déjà été soulevée par Elizabeth Farnsworth et Katherine Allen dans leur travail sur les mères endeuillées ${ }^{13}$ (1996), où elles font le constat d'expériences de marginalisation rapportées par toutes les mères enquêtées, que ce soit au niveau individuel, conjugal ou familial.

Et après $y$ a des gens qui nous sont aussi très proches et qui... ont plus osé nous approcher parce qu'ils savaient pas, ils savaient plus comment, ils avaient plus le droit de nous faire rire, si ils avaient le droit de parler de quelque chose de, pfff... de, de, comment non dit ? Des choses futiles, voilà. Ils arrivaient pas à se situer. Et du coup ils ont préféré mettre de la distance dans nos relations, dans nos rapports. [...] Et du coup plutôt que de faire des gaffes ils se sont mis en retrait, mais vraiment en retrait. [...] Du coup, du coup voilà. II y en a pas beaucoup qui sont passés en travers les mailles du filet. (Christine Raffalli, fils décédé en 2004, à 20 ans, d'un accident de plongée, entretien réalisé en 2014)

Ces expériences d'exclusion, qui sont également des expériences de mise à la marge par rapport aux anciennes sphères de sociabilité, renvoient implicitement à des représentations du deuil étudiées depuis assez longtemps par l'anthropologie. Ainsi Robert Hertz (1906) analyse longuement les temporalités similaires entre les doubles funérailles dans les sociétés traditionnelles indonésiennes et l'exclusion sociale que connaissent les membres de la parentèle du ou de la défunt.e :

Quant aux parents du mort, ils ressentent dans leur personne le coup qui a frappé l'un des leurs : un ban pèse sur eux qui les sépare du reste de la communauté. [...] Par suite de la contagion funèbre, ils sont changés et mis à part du reste des hommes : ils ne peuvent donc plus continuer à vivre comme les autres. (Hertz, $1906: 25)$

L'ambiguïté statutaire évoquée précédemment ne vient donc pas uniquement d'une incertitude normative autour du rôle parental, mais également d'éléments plus relationnels liés à une peur de la contagion. La souillure de la mort toucherait donc les vivants à un niveau symbolique, liant ainsi le statut du/de la défunt·e avec celui de ses proches. La liminalité du statut parental et sa permanence dans le temps sont ainsi liées au statut de l'enfant décédé·e, à sa « présence/absence » constante et à l'incertitude concernant désormais sa place dans la configuration familiale.

Le statut de parent deuilleur·se renvoie donc une liminalité cristallisée : les parents en deuil sont bien dans un " entre deux », et non dans un nouveau statut qu'il s'agirait de définir. L'attention mise à " performer » la parentalité dans un contexte de deuil montre leur volonté de s'y maintenir, et ce malgré la peur de la contamination symbolique qu'ils subissent et qui tend à les exclure de leurs anciennes sphères de sociabilité. La liminalité de la parentalité en deuil s'articule ainsi à la position de l'enfant défunt·e, toujours à la frontière de ce qui fait la famille nucléaire. Cette inscription de l'enfant

\footnotetext{
${ }^{13}$ Leur étude porte sur les mères ayant perdu un·e très jeune enfant, âgé·e de deux jours à qua-
} torze mois. 
décédé·e dans la quotidienneté n'est cependant pas sans être une source de tensions au sein de la famille. La façon dont celle-ci se reconfigure après le décès et la place alors accordée à l'absent.e permettent de renseigner sur ce qui forme désormais les nouvelles frontières familiales.

\section{L'enfant défunt $\cdot e$ et les frontières familiales}

Penser la marginalité parentale ne peut se faire qu'en réfléchissant à la spécificité de la position de l'enfant défunt·e et son inclusion dans la sphère familiale et dans la parenté. Les traces matérielles et les discours entourant la personne décédée peuvent jouer le rôle d'indicateurs pour définir la place qui lui est laissée. L'intégration ou la distanciation de la personne défunte par la valorisation des photos ou des histoires nous informe sur les relations familiales et sur ce que les membres décident d'intégrer ou non dans leur représentation de la famille.

L'attention est ici portée sur les trois dimensions qui forment la frontière familiale, en analysant à chaque fois la " place » qui est laissée à l'enfant décédé·e : la dimension physique et spatiale tout d'abord, avec l'analyse des photographies, la dimension symbolique ensuite, avec la quête des signes, et enfin une dimension plus relationnelle, montrant comment les enfants, même défunt·e·s, continuent d'agir sur les frontières familiales. L'enfant défunt·e étant à la fois inclus.e et exclu·e sur ces trois dimensions (physiques, symboliques et relationnelles), on lui fait franchir en permanence les frontières familiales, les rendant ainsi plus visibles.

\subsection{Rendre le/la défunt·e présent·e : la place des photos}

Après le décès, la famille se réfléchit en définissant des symboles permettant de matérialiser la présence-absence de la personne défunte. Si l'exemple de la chambre à coucher permet bien d'entretenir un rapport de quotidienneté, la présence (ou l'absence) de photographies illustre à la fois la façon dont l'enfant défunt·e est inscrit·e dans des rapports de filiation et la place (dans son sens concrètement spatial) qui lui est laissée. Cependant, les représentations de cette parenté diffèrent, parfois au sein de la même famille. La visibilisation de l'enfant défunt.e peut alors devenir source de tensions. De ce point de vue, l'exemple de la famille Anode est particulièrement intéressant.

Moi je la laisse là [la photo de sa fille], mes garçons n'aiment pas hein. Là y avait une grande photo jusqu'à y a deux jours [rires]. Je viens juste de mettre mes petits [la photo de ses petits-enfants]. [...] Parce que lui [son fils] les photos, ça lui faisait sûrement mal de voir que je mettais des photos de Sophie partout. [...] « Maman t'as vu y a Sophie, y a Sophie, y a Sophie. Tiens elle est pas sur les carreaux là ! ». (Josiane Anode, fille décédée à 23 ans en 2002 d'un cancer, entretien réalisé en 2014)

Chez Josiane, la disposition des photographies permet l'inscription de sa fille dans un régime de quotidienneté, lequel on l'a vu est primordial pour la définition de l'identité parentale. Ce faisant, elle continue de marquer sa présence, en lui faisant occuper un 
espace qui est un espace partagé (les photos sont exposées dans le salon/salle à manger familiale). Or, cette inscription spatiale est une source de tensions avec ses fils.

Des photos, oui j'en ai, mais je les ai, je les regarde pas. En fait il y a tellement chez les parents. C'est un peu un musée. Là ça s'est calmé. On leur a fait la remarque. Ils ont bien remplacé par les petits-enfants. (Rémi, frère de Sophie)

Il est intéressant de noter que, dans le discours de Rémi, il y a, non pas complémentarité, mais opposition entre la place, ici au sens spatial, donnée à sa sœur, et celle prise par ses propres enfants. La tension ici, au-delà de la légitimité de la présence de Sophie, est celle des frontières familiales : qui est inclus·e et qui est exclu·e de ce qui fait, à ce moment-là, la famille ? Le remplacement de ces photos par celles des petits-enfants est en ce sens significatif : l'extension de la parenté doit trouver une matérialisation qui rentre en concurrence avec la visibilisation de la sœur décédée.

\subsection{Les signes ou l'inscription dans la contemporanéité}

Au-delà de la dimension spatiale, des objets et des photographies qui matérialisent de manière métonymique la présence, les parents mobilisent d'autres moyens pour inscrire leur enfant défunt·e dans leur quotidienneté. Il s'agit de s'opposer à l'absence physique en maintenant une présence, au moins symbolique. Le repérage de signes et leur valorisation relèvent de cette stratégie de visibilisation d'une présence. Le maintien d'une forme de relation, via les signes, permet alors d'inclure symboliquement l'enfant dans le cercle familial.

En effet, tous les parents enquêtéee-s déclarent voir ou avoir vu des signes de leur enfant après sa mort ${ }^{14}$. Ceux-ci peuvent prendre différentes formes et être reçus avec ou sans médiation (visite à un·e médium par exemple). Ainsi, la forme d'un nuage, un objet retrouvé ou juste une pensée sont autant de façon de marquer un rappel à l'enfant absent·e.

Je, j'ai l'impression que, souvent je, j'ai l'impression que je l'ai en moi. Quand je vois quelque chose, quand... D'ailleurs ça passe un peu. C'est bizarre ça. II y a des va-et-vient dans les ressentis, dans les... Combien de fois j'ai, en regardant un paysage je dis «Merci Jean, merci. De m'envoyer ces belles images». Tu vois quand il m'arrive quelque chose de bien, dans ma tête je dis « Merci Jean, merci ». (Christine Raffalli, fils décédé en 2014, à 20 ans, d'un accident de plongée, entretien réalisé en 2014)

De manière analogue, l'inscription dans la quotidienneté peut se faire à travers les rêves qui sont vécus et exprimés comme autant de visites de l'enfant.

Enquêtrice : Et, d'ailleurs, est-ce que vous, je peux demander maintenant, est-ce que ça vous a, est-ce que ça vous est arrivé ou est-ce que ça vous arrive encore de... de lui parler?

Pascal Papadopoulos (fils décédé à 16 ans en 2001 d'un cancer, entretien réalisé en 2016) : Parler absolument pas, rêver oui.

${ }^{14}$ Cette mise en avant d'une « présence » après la mort n'est paradoxalement pas liée à une quelconque croyance religieuse. En effet la question des « signes » est généralement bien distinguée, dans les discours, de celle d'une croyance de la vie après la mort (Jégat, 2015). 
Enquêtrice : Rêver.

Pascal Papadopoulos: Rêver, 'fin d'avoir des discussions qui continuent en rêve oui. Mais c'est devenu des rêves... ça devient des rêves qui deviennent incohérents. C'est-à-dire que c'est toujours un visage de gamin mais des situations d'adulte. [...] Je rêve en fait assez souvent en fait. J'arrive, une fois par quinzaine j'ai un petit flash dans un rêve. [...] Mais c'est très aussi contextualisé. C'est dans la vie quotidienne. C'est du maintenant quoi.

Ces expériences partagées renvoient à l'inscription des enfants absent·e s dans une certaine présence et donc au maintien d'une relation, ne serait-ce qu'au niveau symbolique. L'attribution d'un sens particulier à une sensation ou à un évènement, relié.e directement ou indirectement à l'enfant, est une façon de l'inscrire, non seulement dans une quotidienneté, mais également dans une contemporanéité : il/elle est « encore » là, présent.e et actif.ve, puisque, à l'image des signes produits, il/elle peut « surgir ». Cette forme d'inscription temporelle n'est de fait pas que discursive et permet de donner un pouvoir d'action aux personnes disparues (Despret, 2015). La présence des signes et des rêves indique donc, de manière symbolique, l'inscription de l'enfant dans des relations familiales quotidiennes, lui permettant ainsi de perdurer dans le cercle de la parenté.

\subsection{Les modifications des frontières de la parenté}

On l'a vu, le décès de l'enfant modifie la composition de la famille (entendue ici au sens de famille nucléaire) et les représentations que ses membres en ont. L'exemple des photos montre que cette (re)définition peut même être objet de tensions entre les parents et les enfants encore en vie. Mais, au-delà de la place accordée à l'enfant défunt·e dans la configuration familiale, le décès produit un deuxième type de modification des frontières de la parenté : dans certains contextes, elle questionne la validité même de ces frontières en posant la question de l'intégration de personnes qui, au départ, lui étaient extérieures.

Enquêtrice : Et est-ce que tu peux me décrire du coup ta famille ? Des gens que tu considères être, faisant partie de ta famille?

Florian (sœur décédée à 20 ans en 2013 d'un cancer, entretien réalisé en 2015) :

[...] Tracer un... tracer un cercle c'est dur, par contre, 'fin... [long silence ; rires] C'est, c'est pas évident. [...] Après, y a encore une autre dimension. C'est au sein de la famille, justement... 'fin la famille restreinte. 'fin, restreinte... D'inclure ou non... Comment dire. Les copains de mes sœurs. [...] Léa [sa sœur] était quand même très engagée dans sa relation avec Daniel, on a dû déjà beaucoup t'en parler. Et c'est vrai que les, les derniers mois... de sa vie. Et, plus particulièrement la dernière semaine, on a vraiment vécu, vécu ensemble. Et... 'fin c'était un peu une semaine hors du temps. Et quand on est sorti, ouais il était quasiment... 'fin, je sais pas si je le considèrerai, oui comme un frère aujourd'hui. Parce que... le terme est pas forcément le plus approprié. Mais ça, ça s'en approche. Donc... Donc là encore les limites sont pas forcément évidentes.

Cette question des frontières et de leur indéfinition est très bien exprimée par Florian et par ses hésitations. Ici les frontières familiales, et notamment celles de la famille 
nucléaire, sont brouillées par l'inclusion d'une personne extérieure dont le lien affinitaire et électif avec la personne défunte justifierait de l'inclure dans la cellule familiale. Mais cette proximité affective se double ici d'une proximité d'expérience : le fait que le conjoint ait accompagné la sœur dans la mort justifie l'interrogation autour de son intégration dans la nouvelle définition de la famille. Dans d'autres configurations familiales, les ancien·ne·s conjoint·e·s de l'enfant défunt·e continuent ainsi de fréquenter et de garder des contacts avec la famille. Sans que cela ne soit ni la norme ni automatique, l'intégration des ancien·ne·s conjoint·e-s illustre une des manières dont l'enfant défunt·e peut encore agir, au niveau symbolique, sur les frontières familiales.

L'attention portée à ces trois dimensions, physiques, symboliques et relationnelles, permet d'illustrer la façon dont l'absence/présence de l'enfant défunt·e définit et redéfinit des frontières familiales. Ces frontières sont à la fois celles véhiculées par les représentations de ses membres, et font en ce sens l'objet de négociations, et à la fois celles qui sont réaffirmées par des pratiques, notamment par des pratiques d'inscription de l'enfant dans la quotidienneté. La dimension relationnelle notamment, permet de voir comment l'enfant, même défunt·e, continue d'agir et de modifier les frontières familiales et donc de se maintenir dans une « certaine existence»(Despret, 2015: 10-11).

\section{Conclusion}

Cet article tend à montrer comment les concepts de frontières et de marges trouvent une opérationnalité dans des contextes contemporains de deuil. Définir le deuil comme un statut spécifique permet de mettre en relation deux statuts à priori contradictoires (ou du moins peu pensés dans la sociologie française contemporaine) : celui de parents et celui de deuilleur.se.s. Le terme de « marges » renvoie alors à une « mise à la marge de la parentalité », entre parents et non-parents. Mais, plus que marginale, leur position est avant tout liminale : les parents endeuillé·e·s sont exclu·e.s de la parentalité classique mais tendent à s'y réinscrire. Cette réinscription se fait alors notamment au travers de pratiques de soin qui tendent à inscrire la relation avec l'enfant défunt.e dans un registre de quotidienneté. Mais cette relation quotidienne renforce leur marginalisation et leur exclusion des anciennes sphères de sociabilité. C'est là que se trouve peut-être la principale explication de la position liminale de ces parents : dans la présence/absence constante (et réaffirmée par les pratiques) de l'enfant défunt·e.

Cependant, ces dernier.e.s sont en permanence dans l'inclusion/l'exclusion du groupe familial (et notamment de la famille nucléaire). En fonction des positions dans la configuration familiale (membres de la fratrie ou parents), la visibilisation $\mathrm{du} / \mathrm{de}$ la défunt.e et la matérialisation du deuil seront une source de discussions, voire de tensions. Ces tensions sont le signe que, au-delà des situations émotionnelles vécues, la perte d'un.e enfant est toujours un moment de reconfiguration, de redéfinition des frontières familiales, ce concept trouvant ici une toute nouvelle opérationnalité. En ce sens les défunt.e.s franchissent et rendent ainsi visibles les frontières familiales, que ce soit au niveau physique et spatial, symbolique, ou relationnel. 
L'analyse du deuil d'un.e enfant adolescent·e montre ainsi la pertinence et l'actualité des concepts anthropologiques classiques. L'articulation marquée entre un statut défini comme marginal, et qui persiste dans sa marginalité, et l'indéfinition des frontières familiales invite alors à repenser ces concepts à l'aune de terrains plus contemporains et pourtant encore relativement peu travaillés par la sociologie actuelle française.

\section{Bibliographie}

Ariès P. (1977), L'homme devant la mort, Paris, Le Seuil.

BacQué M.-F., Hanus M. (2009), Le deuil, Paris, PUF (« Que sais-je ?»).

Clavandier G., Charrier P., Gourdon V., Pranchere N.S., Rollet C. (2018), Morts avant de naître, la mort périnatale, Tours, Presses universitaires François Rabelais.

DéchauX J.-H. (2014), « Le sacre de l'enfant. Regards sur une passion contemporaine », Revue française de sociologie, vol. 55, n 3, p. 537-561.

Despret V. (2015), Au bonheur des morts : récits de ceux qui restent, Paris, Les Empêcheurs de penser en rond.

FARnsworth E.B., Allen K.R. (1996), " Mothers' Bereavement: Experiences of Marginalization, Stories of Change », Family Relations, vol. 45, n 4, p. 360-367.

Gennep A. Van (2011 [1909]), Les rites de passage : Étude systématique des rites, Paris, Éditions A\&J Picard.

GiRAud A.-S. (2015), « Les "péri-parents” : à la recherche d'un statut spécifique après une mort périnatale », Recherches familiales, $\mathrm{n}^{\circ} 12$, p. 85-97.

Grossetti M. (2009), "Imprévisibilités et irréversibilités : les composantes des bifurcations », in M. Grossetti, M. Bessin, C. Bidart (dir.), Bifurcations, Paris, La Découverte, p. 147-159.

HeRTz R. (1906), « Contribution à une étude des représentations collectives de la mort », L’Année sociologique, vol. 10, p. 48-137.

Hochschild A.R. (2017 [1983]), Le prix des sentiments : au cœur du travail émotionnel, Paris, La Découverte (« Laboratoire des sciences sociales »).

JÉGAT L. (2015), « Perdre un enfant. Une sociologie du deuil à travers les trajectoires individuelles et familiales », Dossiers d'études, nº 175, Paris, CAF.

JÉGAT L. (2019), « Parents en deuil : Maternités et paternités face à la perte d’un enfant », Encyclo, à paraître.

Jeudy H.-P., BAudry P. (2001), Le deuil impossible, Paris, Eshel Éditions.

JoNAS I. (2006), « Un nouveau travail de "care” conjugal : la femme "thérapeute" du couple », Recherches familiales, $n^{\circ}$ 3, p. 38-46.

Julier-Costes M. (2013), « Le monde des morts chez les jeunes », Études sur la mort, vol.142, $\mathrm{n}^{\circ} 2$, p. 125-144. 
Julliard V., Georges F. (2018), « Produire le mort », Réseaux, vol. n² 210, n 4, p. 89-116.

LE GALÈs M. (2017), Ça ne se dit pas ou l'absence d'un mot, Saint-Denis, Édilivre.

Lima L. (2015), Pauvres jeunes : enquête au cour de la politique sociale de jeunesse, Nîmes, Champ Social («Questions de société »).

Memmi D. (2014), La revanche de la chair : Essai sur les nouveaux supports de l'identité, Paris, Éditions du Seuil.

Oria N., Camus J. (2012), " Avoir un premier enfant : un rite d'institution », Recherches familiales, vol. 9, $\mathrm{n}^{\circ}$ 1, p. 49-59.

Régnier-Loilier A., Perron Z. (2016), «Intentions de fécondité et arrivée du premier enfant », Actes de la recherche en sciences sociales, $n^{\circ} 214$, p. 81-93.

Ricceur P (2013 ([1994]), " La souffrance n'est pas la douleur », dans Marin C. (dir.), Souffrance et douleur. Autour de Paul Ricœur, Paris, Presses Universitaires de France («Questions de soin »), p. 13-34.

Roudaut K. (2012), Ceux qui restent. Une sociologie du deuil, Rennes, Presses universitaires de Rennes ( Le Sens social »).

Stiker H.-J. (2007), « Pour une nouvelle théorie du handicap. », Champ psychosomatique, vol. $45, \mathrm{n}^{\circ} 1, \mathrm{p} .7-23$.

TURNER V. (1969), The ritual process: structure and anti-structure, Ithaca, N.Y, Cornell University Pres.

VAN de Velde C. (2015), Sociologie des âges de la vie, Paris, A. Colin.

Zelizer V.A. (1994 [1985]), Pricing the Priceless Child - The Changing Social Value of Children, Princeton, N.J, Princeton University Press. 